\title{
INVESTTIGACIÓN DIDACTICA
}

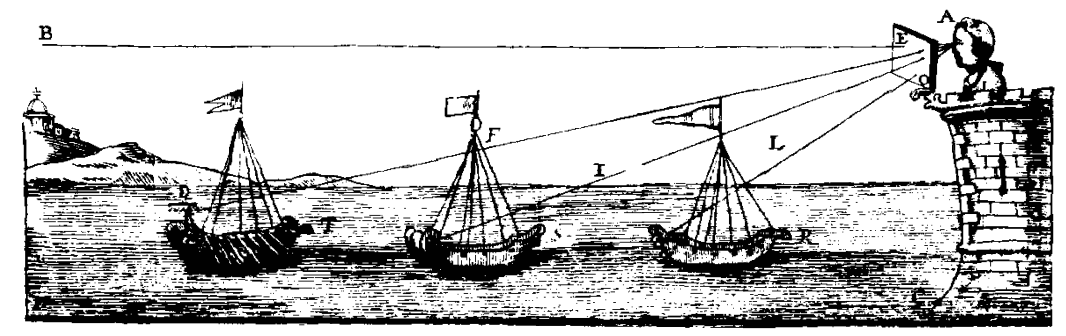

\section{CONDICIONES PARA UN APRENDIZAJE DE CALIDAD EN LA ENSEÑANZA DE LAS CIENCIAS. REFLEXIONES A PARTIR DEL PROYECTO PEEL*}

WHITE, RICHARD, T.

Monash University. Melbourne.

\section{SUMMARY}

In this summary of the topics presented in the closing lecture of the Congress, some of the key-ideas which from our view characterize the science quality learning are reviewed. These ideas have been developed in the framework of the PEEL, an investigation-action project in which teachers and researchers have collaborated.

\section{¿QUÉ ES UN APRENDIZAJE DE CALIDAD?}

Creíamos en mi país, tiempo atrás, que un buen aprendizaje consistía en ser capaz de pasar los exámenes. En el mejor de los casos, los exámenes estaban formados por problemas imaginativos como los de la figura 1 . No hay 
nada erróneo en este tipo de preguntas, pero por sí mismas no proporcionan una prueba válida de comprensión.

Figura 1

Ejemplos de preguntas de examen de física.

\begin{abstract}
Un fabricante produce dos tipos de pilas, A y B. Cada una de ellas se conecta a una resistencia de $100 \Omega$, y se mide durante un tiempo el voltaje resultante. El gráfico muestra el voltaje resultante de cada pila en función del tiempo.
\end{abstract}

Pregunta 6.1

¿Cuál es la potencia de la pila A cuando el tiempo $\mathrm{t}=0$ horas?

Pregunta 6.2

¿Cuál es la energía total proporcionada por la pila A, expresada en W/hora?

\section{Pregunta 6.3}

Un aparato de radio tiene una resistencia efectiva de $100 \Omega$. Funciona solamente mientras su pila le suministra una potencia de por lo menos $0,04 \mathrm{~W}$.

¿Cuanto tiempo funcionará el aparato usando:

a) la pila A?

b) la pila B?

Nuestra creencia de que los exámenes nos lo dicen todo acerca del aprendizaje fue puesta a prueba en los trabajos de investigadores como Za' rour (1976) (Driver, 1983; Driver, Guesne y Tiberghien, 1985; Osborne y Gilbert, 1980; Osborne y Freyberg, 1985), Gunstone y yo mismo (Gunstone y White, 1981) y muchos otros cuyas investigaciones han compilado Pfundt y Duit (1994) en una extensa bibliografía. Estos trabajos revelan que los estudiantes que han superado exámenes formales de ciencias carecen a menudo de una verdadera comprensión de los fenómenos naturales y de las explicaciones de los científicos. Sus creencias pueden no concordar con lo que dicen en los exámenes o con las afirmaciones que aceptan superficialmente en la clase. Un ejemplo de ello es la explicación que varios estudiantes universitarios de física dieron a Gunstone y White (1981) sobre la aceleración de los objetos que caen: «[...] la gravedad aumenta al acercarse a la superficie de la tierra, de manera que los objetos al caer adquieren mayor fuerza y en consecuencia se mueven más deprisa». Brumby (1979) da otro ejemplo: unos estudiantes de medicina que han adquirido conocimientos acerca de la selección natural creen todavía que el color de la piel de una familia cambiaría en una o dos generaciones si se desplazasen a un clima diferente.

Un aprendizaje de calidad implica comprensión profunda y consistencia en las ideas. Cuando ha habido un esfuerzo para resolver las contradicciones, y éstas se han resuelto, entonces las ideas son consistentes. Si ésto es así, las personas no piensan una cosa en un contexto, por ejemplo la clase, y otra diferente en otro contexto, por ejemplo el mundo exterior a la escuela. Las explicaciones oídas a los profesores, o leídas en los libros de texto, compiten y se comparan con las ideas anteriores, y éstas pueden modificarse o descartarse a fin de conseguir la consistencia.

La comprensión requiere algo más que consistencia. No debe haber contradicciones en el conocimiento de una persona, porque dicho conocimiento es limitado. La comprensión requiere un conocimiento extenso. Pero la extensión tampoco es suficiente. El conocimiento debe estar conectado dentro de cada tema, entre temas diferentes, y con las experiencias y aplicaciones fuera de la escuela. Así, la comprensión que los estudiantes tienen de las leyes de los gases aumenta a medida que perciben las relaciones de dichas leyes con la teoría cinética, la noción de molécula, los choques elásticos, el movimiento browniano, la medida de la temperatura y con sus propias experiencias hinchando globos y ruedas de bicicleta o viendo cómo trabajan los taladros neumáticos.

\section{EL PROYECTO PEEL}

El proyecto para aumentar el aprendizaje efectivo, citado también con su acrónimo PEEL (Project for Enhancing Effective Learning) fue un trabajo iniciado en el año 1985. No se trata de un proyecto de investigación, sino de un intento de poner en práctica, en escuelas ordinarias, unos principios derivados de la investigación. No fueron investigadores, sino profesores en activo, quienes, después de conocer las investigaciones sobre metaaprendizaje, tomaron la iniciativa de aplicar dichas investigaciones y modificar su propia práctica.

PEEL involucra profesores de ciencias, pero no se limita a ellos. El protagonista principal es un profesor de ciencias, Ian Mitchell, que estaba preocupado hacía tiempo por la calidad del aprendizaje de sus alumnos. Mitchell obtuvo la cooperación de una docena de colegas de su escuela para poner en práctica los principios del metaaprendizaje, de los que tenía noticia a través de 
John Baird, investigador que antes había sido profesor de ciencias. El proyecto comenzó en la escuela en la que Mitchell trabajaba en 1985. Es importante resaltar que esta escuela no tenía nada de especial. Se encuentra en un suburbio de Melbourne, en una zona de clase trabajadora industrial, y no tiene ventajas de plantilla ni de presupuesto. Hasta que el proyecto empezó, no había nada en los antecedentes de los profesores que se pudiese señalar como excepcional.

En el primer año del proyecto, los profesores describieron la experiencia en un libro editado por Baird y Mitchell (1986). Actualmente PEEL se ha extendido a unas cuarenta escuelas de Australia y, cosa bastante sorprendente, a más de un centenar de escuelas en Suecia, donde se conoce como PLAN, así como en Dinamarca. Su desarrollo en los países escandinavos fue resultado de los esfuerzos de un profesor de ciencias sueco, Sven-Olaf Hagglund, quien leyó el libro de Baird y Mitchell y se desplazó a Australia para conocer mejor el proyecto. Por todo ello se puede afirmar que las personas individuales pueden marcar la diferencia entre que se lleven a cabo innovaciones o no.

Como veremos, el objetivo principal del PEEL era ayudar a los estudiantes a sentirse más motivados y capaces para dirigir y controlar su propio aprendizaje. Hay dos aproximaciones en la investigación didáctica orientadas a este objetivo. Una de ellas se propone el aprendizaje de determinadas técnicas y habilidades de tipo general sin vincularlas al aprendizaje de un contenido específico. Nosotros optamos por la segunda vía, en la que los estudiantes aprenden a orientar su personal forma de aprender al mismo tiempo que aprenden ciencias.

El método de trabajo utilizado por los participantes en el proyecto, lo llamamos «investigación - acción colaborativa». Se basa en procesos cíclicos de «colaboración», «reflexión»y «toma de decisiones». Cada miembro colabora aportando sus puntos de vista, sus conocimientos y sus experiencias. Toda toma de decisiones tiene que estar fundamentada en la teoría, en los conocimientos didácticos conocidos, y debe poderse contrastar con la práctica. La colaboración entre profesores y outsiders ayuda a estimular y guiar el desarrollo individual de todos los participantes, así como el cambio o evolución del propio grupo. Conjuntamente hemos ido desarrollando formas de trabajo en el aula orientadas a favorecer un aprendizaje de calidad.

\section{¿QUÉ CONDICIONES FAVORECEN UN APRENDIZAJE DE CALIDAD?}

La enseñanza, el currículo, el sistema de exámenes y el contexto escolar son factores determinantes para que exista consistencia en las ideas y conexiones amplias en el conocimiento. Pero incluso, cuando dichos factores son favorables, el aprendizaje depende de los estudiantes individuales. Son ellos los que deben optar por aprender de una forma significativa en sus estudios en general, no solamente en el de las ciencias.
Para ello los estudiantes deben tener como propios unos objetivos de aprendizaje. No basta con que el profesor cree las condiciones favorables y marque los objetivos, si el estudiante no los acepta totalmente. Los estudiantes deben hacerse responsables de su aprendizaje y no considerarlo una responsabilidad solamente de sus profesores.

Entre las condiciones que hemos investigado como favorecedoras de un aprendizaje de calidad destacaríamos:

a) la potenciación de la autonomía del alumnado; b) el desarrollo de actidudes y prácticas de cooperación; c) la aplicación de métodos de enseñanza variados; d) la aplicación de métodos de evaluación variados.

\section{Autonomía}

Los estudiantes no se sienten responsables de su aprendizaje si todas las decisiones que se toman en la clase quedan en manos del profesor. Unos y otro deben compartir el control sobre la distribución del tiempo, sobre los temas a tratar, sobre los procedimientos de aprendizaje e incluso sobre la evaluación. Con esto no quiero decir que las clases tengan que ser anárquicas, ni tan sólo democráticas, dejando por lo tanto poco poder al maestro. Lo que propongo es que el equilibrio debe cambiar y que, en lugar de lo que ha sido tradicional en mi país, un control absoluto del profesor y una ausencia de responsabilidad de los estudiantes, debe haber un aprendizaje con objetivos, en el que el maestro ayude a los estudiantes a comprender cada concepto o tema, a través del control que los estudiantes ejercen sobre su propio aprendizaje.

Metaaprendizaje es un término que se refiere al conocimiento que la persona tiene sobre sus propios procesos de aprendizaje, a la conciencia del uso que hace de dichos procesos y a su habilidad para dirigirlos. A estos tres componentes del concepto añado la voluntad de ejercer dicho control y la oportunidad de hacerlo. Son importantes, porque los profesores pueden influir en ellos. Pueden impedir el metaaprendizaje ignorando o penalizando los intentos de los estudiantes de expresar sus propias creencias, o bien ayudarles y destinar una parte del tiempo de clase a discutir las ideas de los estudiantes. Los profesores también tienen un papel promoviendo las habilidades de los alumnos para controlar su aprendizaje, aunque ésta es una cuestión a más largo plazo.

Es probable que en la mayor parte de las aulas australianas los estudiantes perciban que tienen pocas oportunidades de influir en los acontecimientos. En una investigación de Penna, Baird, White y Gunstone (1992), se preguntó a 3.898 estudiantes de 12 a 15 años si les gustaban ciertas características de las clases de ciencias.

Se les planteaban afirmaciones que representaban dos puntos de vista opuestos. Por ejemplo, para una de ellas denominada «Control» en la tabla I, debían escoger 
entre: «Me gusta la manera en que puedo intervenir en decidir lo que vamos a hacer» (que expresaba satisfacción) y «Me gustaría poder decidir más a menudo lo que vamos a hacer» (que se entendía como insatisfacción).

Tabla I

Satisfacción de los estudiantes en diferentes aspectos de las clases de ciencias.

\begin{tabular}{|lllll|}
\hline Porcentaje de satisfechos & & & & \\
\hline Edades & 12 & 13 & 14 & 15 \\
\hline Cantidad de trabajo & 71 & 64 & 63 & 64 \\
Ritmo de trabajo & 73 & 67 & 64 & 62 \\
Dificultad & 68 & 65 & 65 & 68 \\
Importancia & 73 & 67 & 64 & 69 \\
Relación con el mundo & 71 & 63 & 60 & 62 \\
Novedad & 61 & 57 & 55 & 54 \\
Variedad & 52 & 48 & 42 & 41 \\
Control & 44 & 37 & 36 & 37 \\
Actividad mental & 64 & 56 & 47 & 53 \\
Actividad física & 61 & 53 & 48 & 49 \\
\hline
\end{tabular}

(Penna et al., 1992)

En esta tabla cabe observar dos puntos: el descenso de la satisfacción en todos los ítems a medida que avanza la edad, y el hecho de que el control es el aspecto menos satisfactorio.

Paralelamente se preguntó a los estudiantes sus opiniones sobre cuatro aspectos de sus clases de ciencias: si les divertían, si entendían los temas tratados y cuáles eran sus niveles de compromiso y de interés. Para cada aspecto se proponía una escala de respuestas de 1 a 5 . La

Tabla II

Opiniones de los estudiantes sobre aspectos de las clases de ciencias.

\begin{tabular}{|lllll|}
\hline \multicolumn{4}{|l|}{ Porcentaje de estudiantes con opiniones positivas } \\
\hline Edades & 12 & 13 & 14 & 15 \\
\hline Disfrute & 50 & 42 & 35 & 31 \\
Comprensión & 84 & 79 & 72 & 70 \\
Compromiso & 48 & 44 & 38 & 32 \\
Interés & 44 & 36 & 28 & 28 \\
\end{tabular}

(Penna et al., 1992) tabla II muestra los porcentajes de estudiantes que señalan este ítem como uno de los dos mejor valorados. La tabla indica que los cuatro aspectos descienden a lo largo de los años y registran niveles decepcionantes. Las percepciones que tienen los estudiantes sobre la falta de control y sobre su compromiso, interés y disfrute son motivos de preocupación.

Enseñar a los estudiantes a controlar su propio aprendizaje es un objetivo estimulante. Muchos profesores pueden decir que es imposible alcanzarlo, pero el proyecto PEEL ha conseguido un cierto éxito con relación a este propósito.

\section{Cooperación}

Los profesores que han intervenido en PEEL probaron varias vías para animar a sus alumnos a conseguir un aprendizaje reflexivo y con objetivos claros. Algunas de las vías fracasaron, pero los profesores aprendieron mucho de los fracasos, tanto como de los éxitos, y el resultado fue que aparecieron con claridad algunas ideas. Una de ellas es que un aprendizaje de calidad requiere cooperación, más que competición. Los profesores aprendieron a apoyarse unos a otros animándose a probar nuevos métodos, trabajando en equipo, discutiendo frecuentemente las experiencias y participando en las clases de otros profesores. Aprendieron también a apoyar a sus alumnos animándolos a hacer preguntas, a expresar sus creencias o a arriesgar explicaciones sin miedo a parecer estúpidos. Los alumnos aprendieron también a apoyarse unos a otros sin burlarse de los intentos de los demás y tomando en serio todo lo que decían.

\section{Variedad en la enseñanza}

Otra idea clave es que el profesor debe emplear una diversidad de métodos para mantener el interés de los estudiantes. Los resultados del ítem «variedad» en la tabla I muestran que no es fácil ponerla en práctica. Cuando se repetía un procedimiento cualquiera, los estudiantes dejaban de pensar en lo que estaban aprendiendo. Esto es lo que ocurrió al principio de la experiencia, por ejemplo, con los mapas conceptuales (Novak y Gowin, 1984; White y Gunstone, 1992). Al construir sus mapas, al principio los alumnos se esforzaban en pensar, pero en seguida se las ingeniaron para hacerlos de tal manera que, a primera vista, parecían el resultado de la reflexión, pero en realidad eran superficiales. Es natural: los humanos tendemos a automatizar cualquier operación repetida con frecuencia.

Lo que los profesores del PEEL necesitaban no eran respuestas automáticas, sino respuestas meditadas. Para ello empezaron a idear nuevos métodos de clase para que los alumnos tuviesen un aprendizaje más significativo. Hasta ahora los profesores han enumerado 110 métodos, de los cuales Mitchell y Mitchell (1992) han descrito 76, y aparecen otros de vez en cuando en PEEL Seeds, la revista de los profesores. La figura 2 contiene algunos ejemplos breves. 


\section{Relacionar entre sí partes muy diferentes del trabajo}

Éste es un procedimiento muy sencillo y rápido: el profesor selecciona dos partes del trabajo que seguramente los alumnos no han relacionado entre sí, y les pide que lo hagan o bien por escrito, o bien oralmente. Naturalmente, tiene que haber una relación entre ambas partes que valga la pena considerar. Se pueden plantear dos grandes tipos de relaciones. El primero de ellos se refiere a relaciones entre ideas, hechos o conceptos tratados separadamente. Por ejemplo: «iPodéis ver una relación entre las ideas de la página 2 y las de la página 85?» En este caso el profesor supone que no habrán relacionado las ideas de la página 2 con las de la 85, simplemente porque se trataron con varias semanas de distancia. El segundo tipo de relación se refiere a actividades muy diferentes, como el trabajo teórico y el trabajo práctico, o bien el trabajo práctico y los problemas numéricos, o bien excursiones y teoría. Por ejemplo:

- ¿En qué momento del experimento 8 aparece también este aspecto del problema numérico 27 ?

- ¿En qué momento de la excursión vimos una aplicación de la idea de la página 13 ?

Relacionar partes muy diferentes del trabajo requiere pensar de una manera muy parecida a la que se necesita para elaborar un mapa conceptual, pero es más rápido, requiere menos entrenamiento y permite al profesor mantener la atención en un asunto concreto. Ayuda a ejercitar a los alumnos en el pensamiento reflexivo.

\section{Representar una idea}

Este procedimiento tampoco es nuevo, especialmente para los profesores de humanidades. Sin embargo, los profesores de ciencias también pueden aplicarlo, en especial cuando se pide a los estudiantes que representen procesos esencialmente inobservables o complicados. Algunos ejemplos podrían ser: representar las moléculas de perfume que salen de un frasco de perfume al destaparlo, el proceso de reconocer sabores, identificar los electrones en un circuito eléctrico, etc.

\section{Prueba escrita de puntos de vista previos}

En este procedimiento se describen una o más situaciones del mundo real y se hacen preguntas sobre las opiniones corrientes de los estudiantes, que deben responder por escrito.

El profesor recalca que lo importante no es lo que cada estudiante diga, sino la variedad de puntos de vista presentes en la clase. La constatación y resolución de los puntos de vista contrapuestos serán un elemento importante para el trabajo docente subsiguiente. El profesor recoge y resume las respuestas y las relaciona con los tipos de respuesta que se han dado en otras clases, con lo cual legitima el hecho de tener puntos de vista alternativos y da confianza a los alumnos al comprobar que no están solos.

He aquí un ejemplo utilizado en ciencias:

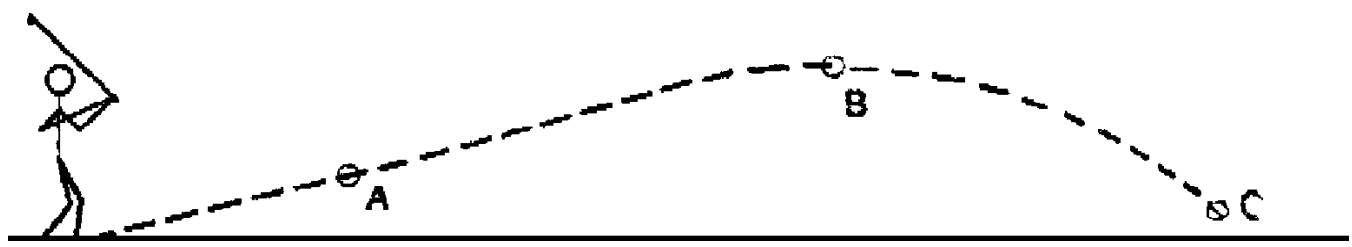

Un jugador de golf lanza una pelota. ¿Cuáles son, en tu opinión, las fuerzas que actúan sobre la pelota después de ser golpeada por el palo de golf?:

a) En el punto A (cuando la pelota está subiendo en el aire)

b) En el punto B (en el punto más alto de su trayectoria)

c) En C (durante el descenso)

Dibuja estas fuerzas mediante flechas y títulos en cada posición.

¿Por qué crees que es así? Razónalo para cada una de las flechas.

Cuando se empieza una prueba de «ideas previas» es importante que los estudiantes no tengan la impresión de que sus puntos de vista van a ser evaluados. Una manera de vencer este temor es pedir que las ideas se den de forma anónima. De todas maneras, hemos comprobado que, cuando los alumnos están habituados a este procedimiento, les gusta «poseer» sus propios puntos de vista. Después, los estudiantes y profesores pueden identificar si han cambiado o ampliado sus ideas, y en qué puntos.

También ha resultado efectivo el uso de diagramas o carteles murales. Por ejemplo, los estudiantes representan los sistemas corporales en una silueta de tamaño natural obtenida resiguiendo el perfil de un estudiante estirado encima de una gran hoja de papel. Mientras se desarrollan estas actividades, se anima a los alumnos a apuntar una lista de las preguntas que han surgido en el debate entre ellos, con objeto de efectuar después lecturas e investigaciones más significativas.

\section{Redefinir palabras habituales}

Este procedimiento trata de conseguir que los estudiantes encuentren significados más precisos a pares de palabras parecidas y de uso corriente, lo cual también ayuda a identificar el grado de comprensión que los estudiantes tienen de los términos del lenguaje cotidiano. 
Colina y montaña, tiempo (meteorológico) y clima son ejemplos del tipo de palabras a las que vale la pena dedicar unos momentos. El procedimiento tiene que ver con la adquisición de conceptos y con la sutileza de significado de los términos nuevos, lo que obliga a los estudiantes a ser precisos con los criterios.

5. Los estudiantes seleccionan sus propios problemas

Este procedimiento da a los alumnos la responsabilidad de selecionar los trabajos que deben hacer en su casa. En su forma más sencilla, el procedimiento consiste en dejar que elijan por sí mismos los problemas. Por ejemplo: «Selecciona los ocho problemas que te parezcan que te serán de mayor ayuda». Para ello se requieren dos habilidades. La primera de ellas, analizar problemas según tipo y dificultad, mediante la cual los estudiantes deben ser capaces de hacer una elección equilibrada. La segunda habilidad es diagnosticar las áreas en que están más necesitados. A veces conviene ayudarles un poco en el primer aspecto, clasificando los problemas por tipos o por niveles de dificultad.

Cuando los estudiantes pueden decidir el número de problemas que deben hacer, se les puede dar más independencia: «Selecciona entre 1 y 10 problemas». Si se les da esta libertad, los alumnos suelen reaccionar con mucha responsabilidad.

Una estrategia parecida incluye dos ejercicios. En el primero de ellos los estudiantes responden una serie de preguntas sobre un tema determinado. El profesor analiza los resultados del ejercicio, selecciona los tipos de pregunta que los alumnos no han contestado bien y escribe algunas preguntas más de cada uno de estos mismos tipos. En el segundo ejercicio se da a los alumnos la nueva lista de preguntas, que no están ordenadas por tipos ni en el mismo orden que en el ejercicio. Los estudiantes deben leer la lista y decir qué preguntas corresponden a los mismos tipos que las del primer ejercicio. A continuación deben mirar sus respuestas del primer ejercicio y decidir en qué tipos de preguntas necesitan más práctica o bien pedir que el profesor amplíe la explicación. Entonces, ellos mismos elaboran una selección de la lista, con un mínimo de una pregunta por cada tipo. Los estudiantes valoran que se les dé este tipo de control. El hecho de tener que decidir sobre tipos y número de preguntas les da un mayor sentido de responsabilidad.

6. Ordenar notas o instrucciones desordenadas

Este procedimiento ayuda a los estudiantes a ordenar de forma lógica las ideas o instrucciones. Se les da un texto, que puede ser un poema, una narración o bien una serie de instrucciones de laboratorio o de recetas, descompuesto en frases o párrafos cuyo orden se ha alterado, para que los reordenen. Una variante avanzada consiste en darles un diagrama sin textos y, aparte, los textos que le corresponden, que deben colocar en su sitio.

Ningún profesor utiliza todos los métodos, porque la variedad excesiva es tan perjudicial como la excesivamente reducida. El profesor debe aprender cómo poner en práctica cada método, y los estudiantes, cómo responder a él.

La variedad es vital para que los estudiantes aprendan a aprender; es decir, para que adquieran estrategias generales, como, por ejemplo, saber separar los puntos importantes de los secundarios en cualquier comunicación o situación, prestando atención a las ambigüedades, manteniendo la atención en la tarea y evaluando el avance. Cada vez que se sigue un procedimiento de enseñanza nuevo se promueve que los estudiantes desarrollen nuevas estrategias de aprendizaje o que practiquen un conjunto diferente de ellas. No ocurre lo mismo si la enseñanza es rutinaria.

\section{Variedad en los sistemas de evaluación}

No es fácil romper con la rutina porque, aunque los alumnos se quejan de aburrimiento, la rutina les exige poco esfuerzo por su parte, mientras que el aprendizaje significativo requiere dedicación. Se les debe mostrar que, si se aburren, es porque no aprenden nada. No saben que no aprenden porque la enseñanza rutinaria está asociada con la rutina en los pruebas de evaluación. Superar unas pruebas rutinarias no es una medida válida de aprendizaje significativo. Cuando se quiere animar a los estudiantes a pensar sobre su aprendizaje, la diversi- dad en las formas de evaluación es tan importante como la diversidad en la enseñanza o en el trabajo de clase.

La investigación sobre concepciones alternativas en ciencias ha generado nuevas formas posibles de evaluar. White y Guinstone (1992) describen algunas de ellas, entre las que se incluyen mapas conceptuales, diagramas relacionales y tareas de predicción - observación -explicación, todas ellas adecuadas para utilizarlas en clase.

Los mapas conceptuales no son muy eficaces para comprobar los conocimientos, pero pueden revelar si el tipo de enlaces que un estudiante ha establecido entre los términos clave es adecuado. La figura 3 muestra mapas realizados por tres estudiantes de 10 años a los que se pidió que presentasen la relaciones entre seis palabras o expresiones: electrones, electricidad estática, corriente eléctrica, átomos, plástico y metal. En el mapa número 1 hay pocos enlaces, y la relación entre electrones y electricidad estática es imprecisa; revela un nivel de comprensión bajo. El mapa 2 tiene más conexiones, pero tres de ellas carecen de explicación. Si se tratase de un olvido, el profesor debería preguntar al estudiante por las explicaciones que faltaban. La relación «en los electrones hay átomos» podría ser una mala interpretación de la noción previa de que «todo está formado por átomos», que el profesor podría comprobar. El mapa 3 tiene más enlaces y éstos están etiquetados razonablemente. La relación de la parte superior afirmando que la electricidad estética y la corriente eléctrica son diferentes requiere también que el profesor haga algunas pre- 
Figura 3

Mapas conceptuales hechos por estudiantes de 10 años (White y Gunstone, 1992).

1

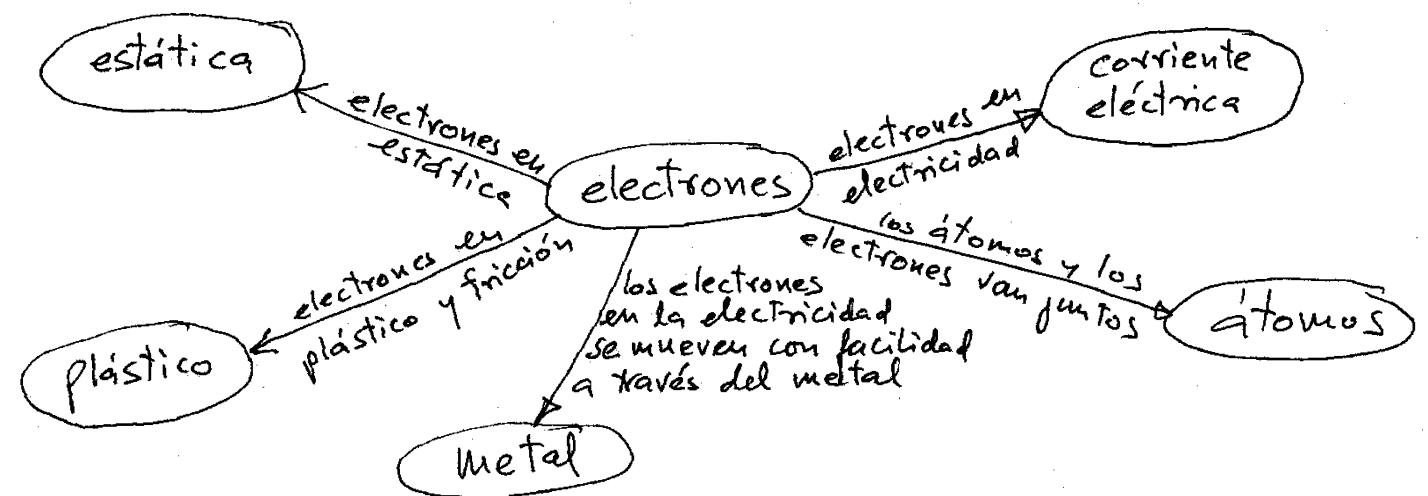

2
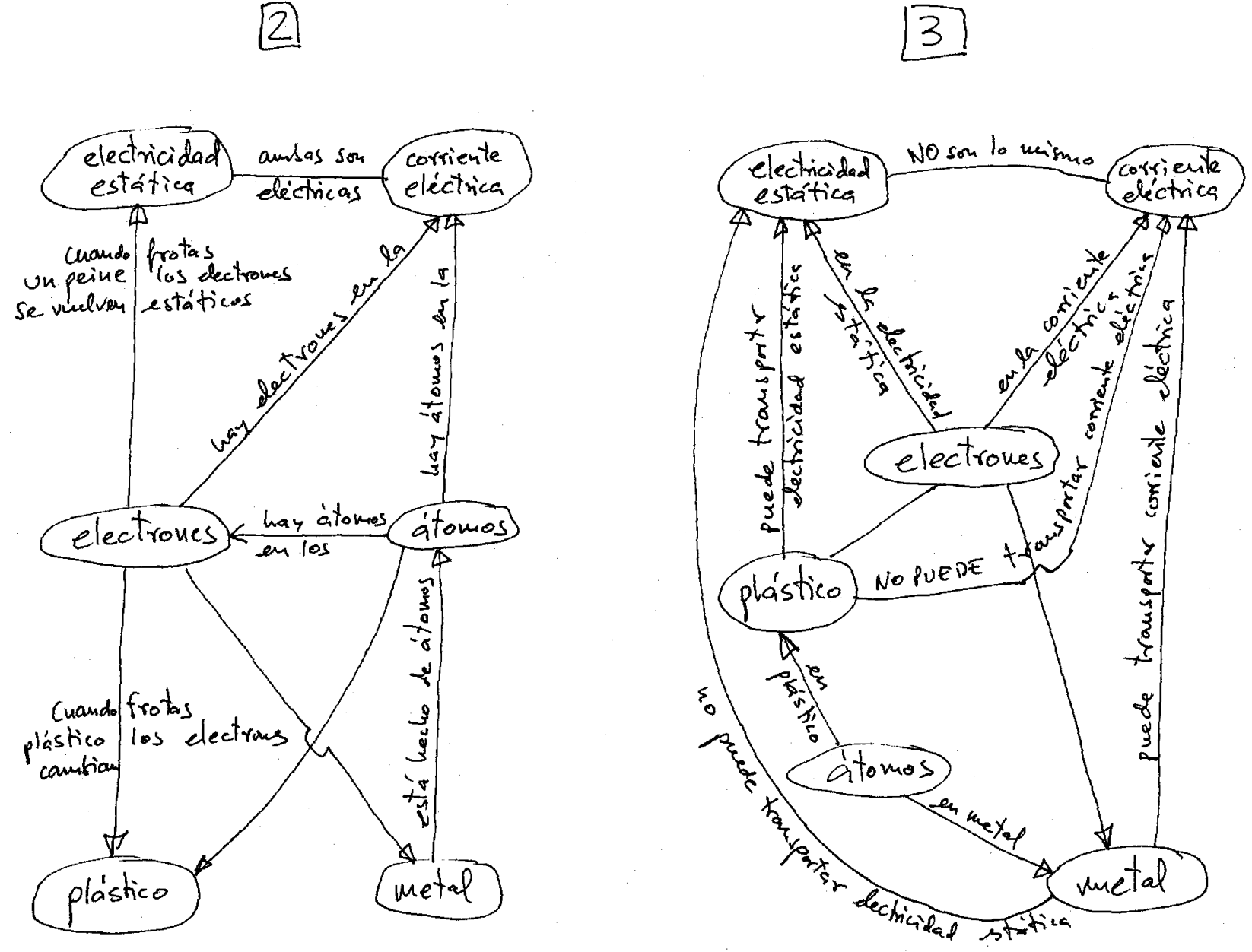
guntas más para establecer qué es lo que el estudiante quiere decir. Un mapa conceptual no da una imagen completa de lo que el estudiante comprende y, como cualquier otra forma de prueba, necesita ser complementada con otras preguntas. Sin embargo, los mapas proporcionan una representación clara de la comprensión.

Las actividades de predicción - observación - explicación son una prueba de comprensión muy poderosa, adecuada al contenido de la ciencia. Los estudiantes deben predecir el resultado de un cambio en una situación física y, sobre todo, razonar su predicción. A continuación observan el cambio, describen lo que ven y explican cualquier discrepancia que se haya observado entre la predicción y la observación. Por ejemplo, en un circuito montado según el diagrama de la figura 4 , los estudiantes deben predecir lo que ocurrirá cuando el interruptor esté abierto.

Figura 4

Diagrama del circuito usado en la actividad POE.

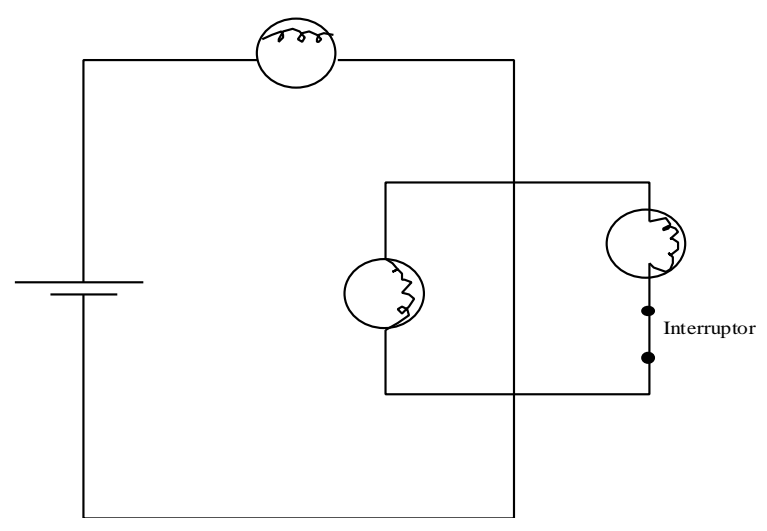

\section{EVIDENCIAS DE UN APRENDIZAJE DE CALIDAD}

Unas respuestas correctas de los estudiantes a preguntas diversas, imaginativas y válidas no son la única forma de comprobar que se ha conseguido el aprendizaje significativo deseado por el profesor; también lo es el comportamiento de los estudiantes en la clase.

Cuando empecé a enseñar, hace cuarenta años, existía una opinión generalizada, que aún persiste, de que una clase silenciosa es una buena clase. Nada hay que objetar si el silencio está acompañado por un aprendizaje serio, en el que se piensa acerca de lo que se enseña. Otra cosa es cuando el silencio refleja apatía o indiferencia. Nadie aboga por el alboroto y por la indisciplina irresponsable. El comportamiento responsable en el aprendizaje, sin embargo, requiere algo más que una recepción pasiva de información.
El hecho de que los estudiantes conducen activamente su propio aprendizaje se traduce en una conducta abierta, por ejemplo, haciendo preguntas reflexivas o avisando cuando no entienden algo. Desde luego, la ausencia de tal conducta no indica necesariamente que los estudiantes no estén comprendiendo. Pero cuando dicha conducta se da, pone en evidencia que los estudiantes están pensando sobre el contenido de la clase, ponderando sus implicaciones y evaluando si concuerdan con sus nuevas creencias.

Los profesores de PEEL desarrollaron una lista de más de veinte «conductas de buen aprendizaje» que aceptan como indicadores de un aprendizaje de calidad.

Dado que las «conductas de buen aprendizaje» son abiertas, es posible contar las veces que se dan en las clases. Hemos comprobado que en las aulas australianas es poco frecuente. Mi impresión después de haber hecho comprobaciones durante un cierto número de años es que es frecuente una cifra menor de diez «conductas de buen aprendizaje» en una clase de 40 minutos. Este dato es consistente con los estudios de interacción en el aula del tipo iniciado por Flanders (1970), en los cuales la mayor parte del tiempo está ocupado por la explicación del profesor. Las clases del PEEL contrastan claramente con este hecho. Una serie de clases de Mitchell con clases del nivel 10 (15 años de edad) daban un promedio de unas 50 «conductas de buen aprendizaje» por clase, más de una por minuto (White y Mitchell, 1993), lo cual revela un estilo diferente de dar clase. El apéndice A recoge una ilustración de este estilo de clase mediante un resumen de una de las clases de Mitchell.

La lista de «conductas de buen aprendizaje» de los profesores del PEEL no pretende ser completa. Está abierta para que pueda ampliarla cualquier profesor o investigador, sugiere muchos estudios posibles y recomiendo usarla en investigación. Una pregunta interesante, por ejemplo, sería: ¿La frecuencia de «conductas de buen aprendizaje» varía con los temas?

Durante mucho tiempo una enseñanza de calidad ha sido un reto para investigadores y profesores, y las tradiciones y métodos de investigación han mantenido a investigadores y profesores separados entre sí, de manera que no se podían ayudar unos a otros para hacer frente a este reto. Los recientes cambios en el estilo de investigación dan una oportunidad para que los profesores puedan realizar una investigación de primer orden en el aula. Se han dado unos primeros pasos en la identificación de concepciones alternativas, en el desarrollo de diversas pruebas de comprensión, en la puesta en práctica de programas como el PEEL para promover el metaaprendizaje, en el diseño de procedimientos de enseñanza innovadores y en la especificación de conductas que indican un buen aprendizaje.

Construyamos sobre estos cimientos y compartamos nuestros nuevos conocimientos con los colegas de aula, $\mathrm{y}$ de esta forma haremos avanzar un aprendizaje de calidad. 
Figura 5

Buenas conductas de aprendizaje.

1. Avisa al profesor cuando no entienden

2. Pregunta al profesor por qué están equivocados

3. Dice al profesor qué es lo que no entienden

4. Compara el trabajo con las instrucciones, corrigiendo errores y omisiones

5. Cuando se bloquea, repasa el trabajo anterior antes de preguntar al profesor

6. Comprueba la comprensión personal de la instrucción y del material. Pide más información si hace falta

7. Busca razones para aspectos del trabajo

8. Anticipa y predice posibles resultados

9. Planifica una estrategia general antes de empezar

10. Explica propósitos y resultados

11. Comprueba el trabajo del profesor para encontrar errores, y propone correcciones

12. Busca conexiones entre ideas y actividades adyacentes

13. Busca conexiones entre ideas y actividades no ady acentes $y$ entre temas diferentes

14. Busca más información de forma independiente, aplicando ideas surgidas en clase

15. Busca conexiones entre ideas, temas diferentes

16. Hace preguntas inquisitivas pero generales

17. Ofrece ejemplos personales relevantes en general

18. Busca conexiones específicas entre el trabajo escolar y la vida personal

19. Trata de descubrir debilidades en la propia comprensión. Comprueba la consistencia de sus explicaciones a través de diversas situaciones

20. Sugiere nuevas actividades y procedimientos alternativos

21. Expresa desacuerdo

22. Propone ideas, nuevas intuiciones y explicaciones alternativas

23. Justifica opiniones

24. Reacciona y se refiere a comentarios de otros estudiantes

25. Cuestiona un texto o una respuesta que el profesor ha sancionado como correctos

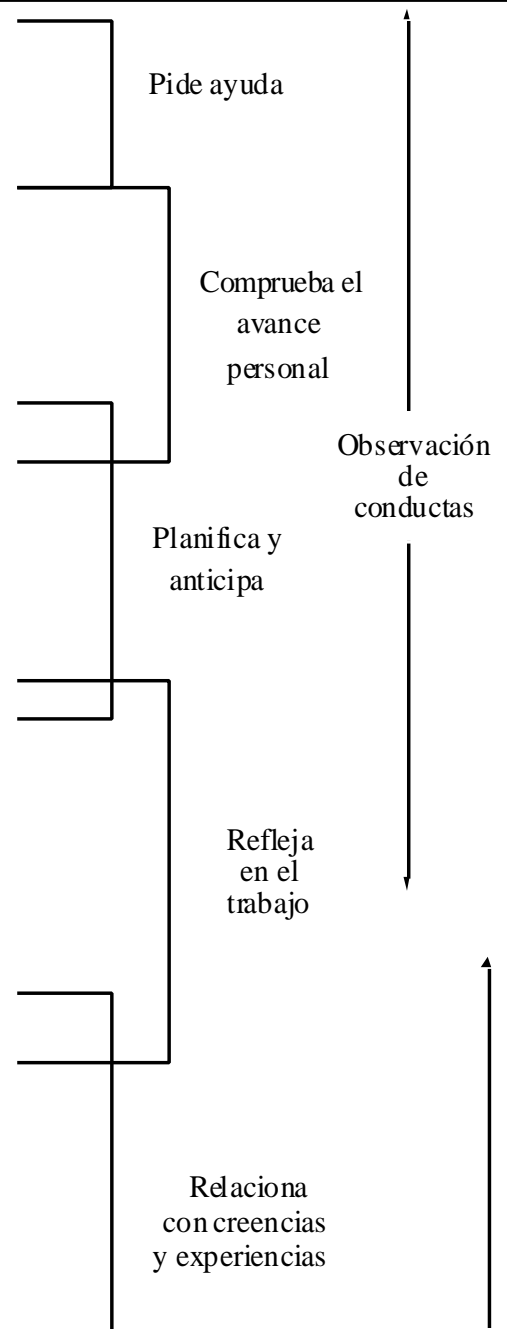

Construcción

y reconstrucción de conductas

Adopta

una postura 


\section{NOTA}

Ponencia presentada en el V Congreso Internacional sobre Investigación en la Didáctica de las Ciencias (Murcia, 10 a 13 de septiembre de 1997). Ha sido traducida del inglés por Carles Martínez.

\section{REFERENCIAS BIBLIOGRÁFICAS}

BAIRD, J.R., MITCHELL, I.J. (eds.) (1986). Improving the quality of teaching and learning. An Australian case study The Peel project. Melbourne: Monash University.

BRUMBY, M. (1979). Problems in learning the concept of natural selection. Journal of Biological Education, 13(2), pp. 119-122.

DRIVER, R. (1983). The pupil as a scientist? Milton Keynes: Open University Press.

DRIVER, R., GUESNE, E. y TIBERGHIEN, A. (1985). Children's ideas in science. Milton Keynes: Open University Press.

GUNSTONE, R.F. y WHITE, R.T. (1981). Understanding of gravity. Science Education, 65, pp. 291-299

MITCHELL, I. (1992). The class level, en Baird,J.R. y Northfield, J.R. (eds.). Learning from the PEEL experience. Melbourne: Monash University Faculty of Education.

MITCHELL, J. y MITCHELL, I. (1992). Some classroom procedures en Baird, J.R. y Northfield, J.R. (eds.). Learning from the PEEL experience. Melbourne: Monash University Faculty of Education.

NOVAK, J.D. y GOWIN, D.B. (1984). Learning how to learn. Cambridge: Cambridge University Press.
OSBORNE, R.J. y FREYBERG, P. (1985). Learning in science: The implications of children's science. Auckland: Heinemann.

OSBORNE, R.J. y GILBERT, J.K. (1980). A method for investigating concept understanding in science. European Journal of Science Education, 2, pp. 311-321.

PENNA, C., BAIRD, J.R., WHITE, R.T. y GUNSTONE, R.F. (1992). A survey of components of challenge and outcomes in science learning. Comunicación a la reunión de la Australasian Science Education Research Education, University of Kiel.

PFUNDT, H. y DUIT, R. (1994). Bibliography: Students' alternative frameworks and science education (4a. ed.). Kiel: Institute for Science Education, University of Kiel.

WHITE, R.T.y GUNSTONE, R.F.(1992). Probing understanding. Londres: Falmer.

WHITW, R.T. y MITCHELL, I.J. (1993). The promotion of good learning behaviours. Comunicación presentada en la conferencia de la European Association for Research on Learning and Instruction, Aix-en-Provence.

ZA'ROUR, G.I. (1976). Interpretation of natural phenomena by Lebanese school children. Science Education, 60, pp. 277-287. 


\section{APÉNDICE A}

Tres minutos extraídos de una lección de PEEL (Mitchell 1992)

Era la cuarta lección de mecánica en el nivel 10A (15 años) en 1987. Mi objetivo a largo plazo, que no esperaba conseguir solamente con esta lección, era ayudar a los estudiantes a reestructurar sus ideas sobre las fuerzas de reacción de acuerdo con la tercera ley del movimiento de Newton: «Para cada acción existe una reacción igual y opuesta.» En la lección precedente, la discusión sobre unas pruebas escritas anteriores habían puesto de manifiesto dos ideas, propuestas inicialmente por los alumnos Katie y Ward, acerca de si una mesa empuja o no hacia arriba un libro puesto encima de la misma. El cuadro IV.1 resume los argumentos de varios estudiantes en apoyo de una u otra de las dos opiniones.

\section{Cuadro IV.1.}

Ideas de Katie y de Ward sobre la fuerza de reacción.

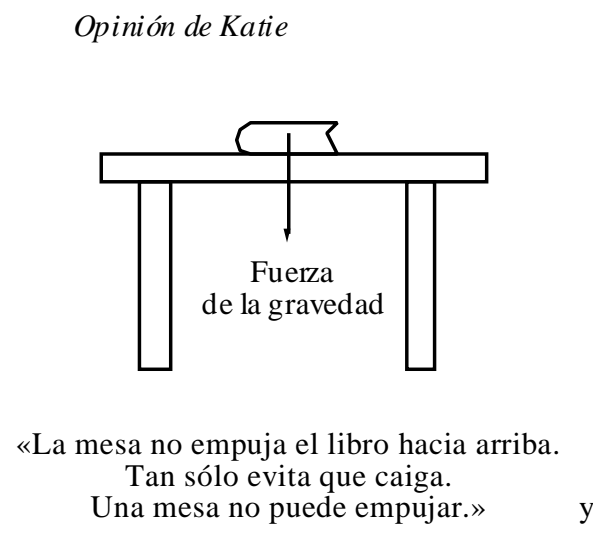

Tan sólo evita que caiga.
Una mesa no puede empujar.»

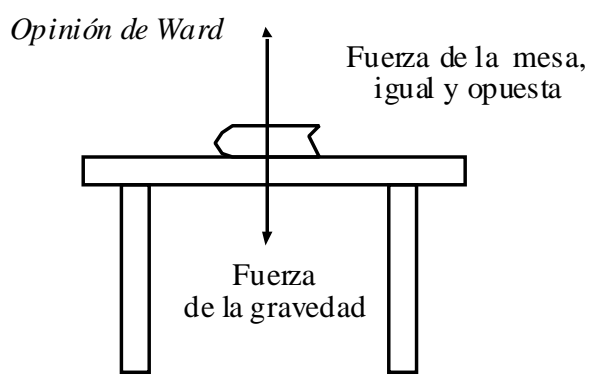

«La mesa tiene que empujar el libro hacia arriba con una fuerza igual y opuesta, porque, si no, el libro se movería.»

He descubierto que la opinión de Katie es la más frecuente siempre que he enseñado este punto. La de Ward es coherente con la de Newton, pero a muchos estudiantes no les convence, porque nadie ha podido explicar cómo puede una mesa ejercer un empuje. La explicación correcta es que, cuando se coloca el libro sobre la mesa, ésta se deforma flexionándose ligeramente hacia abajo, y esta flexión ejerce una fuerza hacia arriba. Pero la mayoría de mesas son demasiado rígidas para que esta flexión sea perceptible.

Quería que los estudiantes pudiesen percibir la flexión de la mesa, de manera que preparé una mesa mucho más flexible, con libros y una regla graduada, como se muestra en el cuadro IV.2

\section{Cuadro IV.2}

Mesa construida con una regla.

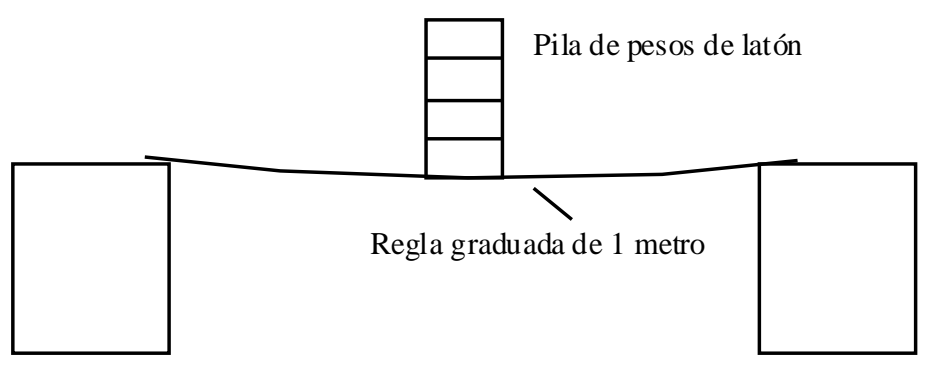

Este dispositivo indujo a varios estudiantes a una tercera opinión, propuesta por Danielle, según el cuadro IV.3. 


\section{Cuadro IV.3}

Opinión de Danielle sobre las fuerzas de reacción.

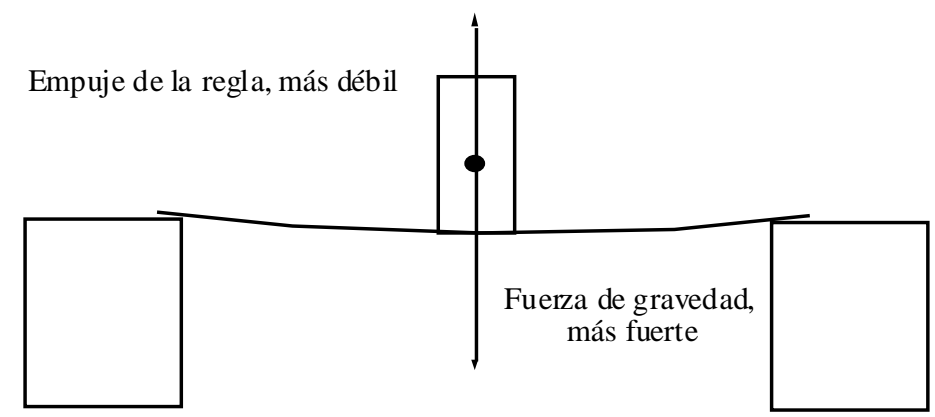

Después de mi resumen de la discusión de la lección anterior, Ward comunica su reflexión:

Ward: La regla empuja hacia arriba. Es como un muelle, porque, cuando le pones encima el peso, es como si hubieses apretado un muelle. Si lo sueltas, saltará otra vez hacia arriba.

(Este comentario podría dar lugar a que la clase discuta cómo puede una mesa empujar hacia arriba. Deseo que lo hagan, pero no quiero revelar mi opinión todavía.)

Profesor: ¿Crees que empuja hacia arriba porque está flexionada?

(Estoy presentando, con neutralidad, lo que veo como el punto nuevo clave de lo que ha dicho Ward.)

Ward: Sí.

Brad: ¿Si estuviese empujando hacia arriba no estaría recta?

[1] (Brad reacciona a las ideas de Ward. Su argumento es lógico: no se puede imaginar que la regla esté empujando hacia arriba y, en cambio, no se mueva. Esta cuestión es central, y quiero que piensen sobre ello. Contesto a Brad de una forma parecida a como lo he hecho con Ward.)

Profesor: Si estuviese empujando hacia arriba, estaría recta... Así, ¿estás diciendo que, como que está flexionada, no empuja hacia arriba?

Brad y Kay: Sí

Ward: (Interrumpiendo.) Tiene que empujar hacia abajo con la misma fuerza con que empuja hacia arriba.

Profesor: Entonces, ¿estás diciendo que la gravedad empuja hacia abajo y la regla lo hace hacia arriba?

Danielle: (Interrumpiendo.) Si estuviese empujando hacia abajo lo mismo que hacia arriba, la regla estaría recta.

(Ahora Danielle introduce una nueva posibilidad. Quiero que todos lo vean claro, de manera que dibujo un diagrama de su punto de vista [Cuadro IV.3] al lado del resumen de los puntos de vista de Ward y Katie dibujados antes. La opinión de Danielle de que tiene que haber alguna fuerza hacia arriba puede ser muy útil para acercarnos a la tercera ley de Newton.)

Profesor: Muy bien; así, el argumento de Danielle es... que las fuerzas no pueden ser iguales y opuestas, porque la regla no estaría curvada.

Danielle: Podría ser una fuerza hacia arriba y otra mayor hacia abajo.

Profesor: Bien. Así estás dispuesta a aceptar que hay una fuerza que empuja hacia arriba, pero tiene que ser menor que la gravedad que empuja hacia abajo. La dibujarías así, ¿no? (El profesor dibuja el esquema del cuadro IV.3.). Estás dispuesta a aceptar esto (Señala una fuerza hacia arriba.) pero, como la regla está flexionada, la fuerza que empuja hacia abajo es mayor.

Ward: $\quad$ Si hay una fuerza mayor hacia abajo, entonces, ¿por qué no se mueve hacia abajo?

Kay y Danielle: (Interrumpiendo.) Pero si lo ha hecho, se ha movido hacia abajo. 


\section{INVESTIGACIÓN DIDÁCTICA}

(Aquí empieza un debate entre los estudiantes en el que no hace falta que intervenga. Los estudiantes se sienten muy implicados e interesados.)

Ward: $\quad$ No, no se mueve; lo que digo es que ahora no se mueve. Si hubiese una fuerza mayor hacia abajo, no pararía de moverse hacia abajo.

Danielle: Pero las dos fuerzas no pueden ser iguales.

Bard: $\quad$ Es como si se añade peso al suelo. No puede ir más abajo.

Ward: ¿Por qué no?

James: $\quad$ Porque no ha conseguido más pesos. (Un comentario no muy útil.)

Ward: $\quad$ Si hay más fuerza empujando hacia abajo, ¿por qué no se puede flexionar?

Brad: $\quad$ Porque es la base, ya lo sabes. Quiero decir que, si apilas los libros en la mesa, no van a empujar la mesa hacia debajo del suelo.

(Brad cree que el suelo rígido no necesita ejercer ninguna fuerza hacia arriba para evitar que los pesos colocados encima suyo se muevan hacia abajo.)

Ward: $\quad$ Lo que pasaría es que la mesa volvería a empujar hacia arriba.

Kay: $\quad$ En cualquier caso, si pones esos pesos en esta mesa (Señala hacia una mesa de la clase.), no se moverán hacia abajo.

Danielle: $\quad$ No, pero si pones cosas más pesadas encima, podría.

(Ward puede estar convenciendo a Kay. Ella ha descubierto una cuestión importante: no habremos conseguido gran cosa mostrando una regla que se dobla bajo un peso, a menos que podamos mostrar que las mesas «de verdad» también se flexionan. Me propongo mostrar esto más tarde haciendo poner a los estudiantes de pie encima de las mesas. No quiero desanimar a Kay, pero debo parar la discusión, que se está complicando demasiado al hablar de dos clases de mesa a la vez. Danielle ha hecho una buena observación. Decido intervenir, dando a entender a Kay que su observación la comentaremos después, porque hay que acabar un tema antes de empezar otro.)

Profesor: Muy bien, después tendremos que volver a este punto y ver qué pasa con esta mesa (la de la clase). Estás diciendo que he enredado un poco la cuestión porque he usado una regla flexible. De acuerdo, volveremos a esta cuestión, tal vez hoy mismo... pero sigamos con la situación de la que estábamos hablando... (la mesa construida con la regla).

Kay: $\quad$ Sí.

(Creo que Kay confía en que me acordaré de volver a su observación.)

Entonces llamé a otros dos estudiantes que apoyaban a Ward. No aportaron argumentos nuevos, de manera que decidí que era el momento de establecer que la regla estaba empujando hacia arriba, por lo menos con cierta fuerza. Pedí a un estudiante, Mark, que saliera y pusiera la mano sobre la regla flexionada mientras yo quitaba los pesos. Dijo que notaba cómo la regla empujaba hacia arriba. La experiencia resultó efectiva: nadie volvió a decir que la regla no ejercía fuerza hacia arriba. 